\title{
Algo más sobre Victor Wolfvoet. Una segunda réplica de la Crucifixión atribuida a Abraham van Diepenbeeck
}

Something else about Victor Wolfvoet. A second replica of the Crucifixion attributed to Abraham van Diepenbeeck

\section{Matías Díaz Padrón ${ }^{1}$}

Real Academia de arqueología de Bélgica

Resumen: En este artículo se restituye a Victor Wolfvoet la autoría de una Crucifixión atribuida a Abraham van Diepenbeeck. Así lo prueban la iconografía y el estilo de esta pintura influenciada por un boceto de Rubens $y$, especialmente, por un grabado de Jacob Neefs con el mismo tema.

Palabras clave: Victor Wolfvoet; Abraham van Diepenbeeck; Peter Paul Rubens; Jacob Neefs; Crucifixión.

Abstract: In this paper the authorship of a Crucifixion attributed to Abraham van Diepenbeeck is returned to Victor Wolfvoet. It is proved by the iconography and the style of this painting, which is influenced by a sketch from Rubens and, specially, by an engraving from Jacob Neefs in which the same theme is depicted.

Key-words: Victor Wolfvoet; Abraham van Diepenbeeck; Peter Paul Rubens; Jacob Neefs; Crucifixion.

e uno al merecido homenaje al profesor Hugo de Schepper con esta pequeña contribución al arte del siglo XVII en Flandes y su área de influencia hispana. Tuve con el profesor De Schepper la ocasión de compartir el acontecimiento de Europalia 85: Splendeur $d$ 'Espagne et des villes belges, 1500-1700 en Bruselas, en el que se contribuyó a destacar el feliz encuentro de España y Flandes en los momentos cruciales de su historia.

\footnotetext{
' http://orcid.org/0000-0002-5137-7583

(c) 2018 Philostrato. Revista de Historia y Arte
} 
Es de interés señalar una Crucifixión sobre tabla de pequeño formato ( $58 \mathrm{x}$ 43,5 cm.) en el mercado artístico de París en 2008, que fue atribuida a Abraham van Diepenbeeck ${ }^{2}$. (Fig. 1) Sin embargo, el tipo de soporte, la composición y la calidad de la pintura coinciden con los del original de Victor Wolfvoet en la colección Gerstenmaier de Madrid ${ }^{3}$; variando sólo en altura éste último pues está dotado de unos centímetros más (64 x 43,5cm) (Fig. 2).

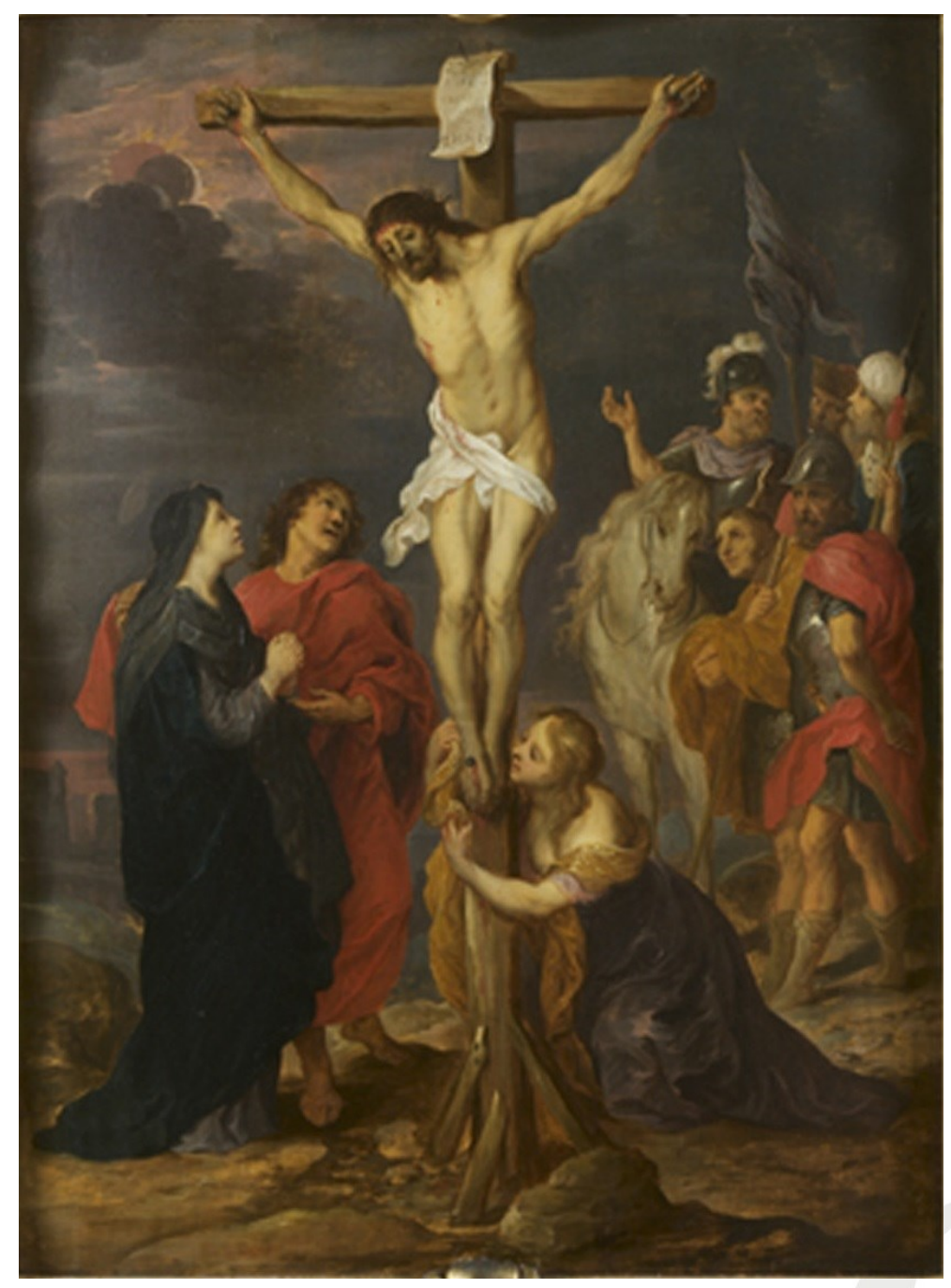

Fig. 1. Victor Wolfvoet, Crucifixión, colección privada

La refracción de la luz en la preparación de estas dos tablas aviva los colores, los cuales lucen con más prestancia que en las repeticiones en cobre, soporte más frecuente en la producción del pintor. Dentro de la evidente

\footnotetext{
2 Tajan París, venta no 8838 (26-VI-2008), no 41; antes en Sotheby's 10-X-2007, no 59 , como anónimo de escuela flamenca.

3 Matías Díaz Padrón, "Un Calvario de Victor Wolfvoet en la colección Gerstenmaier", Tendencias, no 68, (2013), pp. 82-84. Procede de colección privada en Madrid como anónimo seguidor de Rubens: Inmaculada Alonso Blázquez, "Victor Wolfvoet, Calvario" en Colección Hans Rudolf Gerstenmaier. La Pintura Flamenca en la Colección Gerstenmaier, dir. Matías Díaz Padrón y María Toral Oropesa, (Lerner \&Tf Editores, 2017), pp. 82-83.
} 
correspondencia de una y otra obra, en la tabla que tratamos es visible la mayor amplitud espacial en los extremos laterales, respecto a la composición de la colección de Madrid. Así, el grupo de la derecha, formado por soldados a pie y a caballo, está ligeramente desplazado hacia ese mismo lado, mientras que el resto de las figuras mantienen idéntica localización, si bien aumenta el margen lateral izquierdo de la escena.

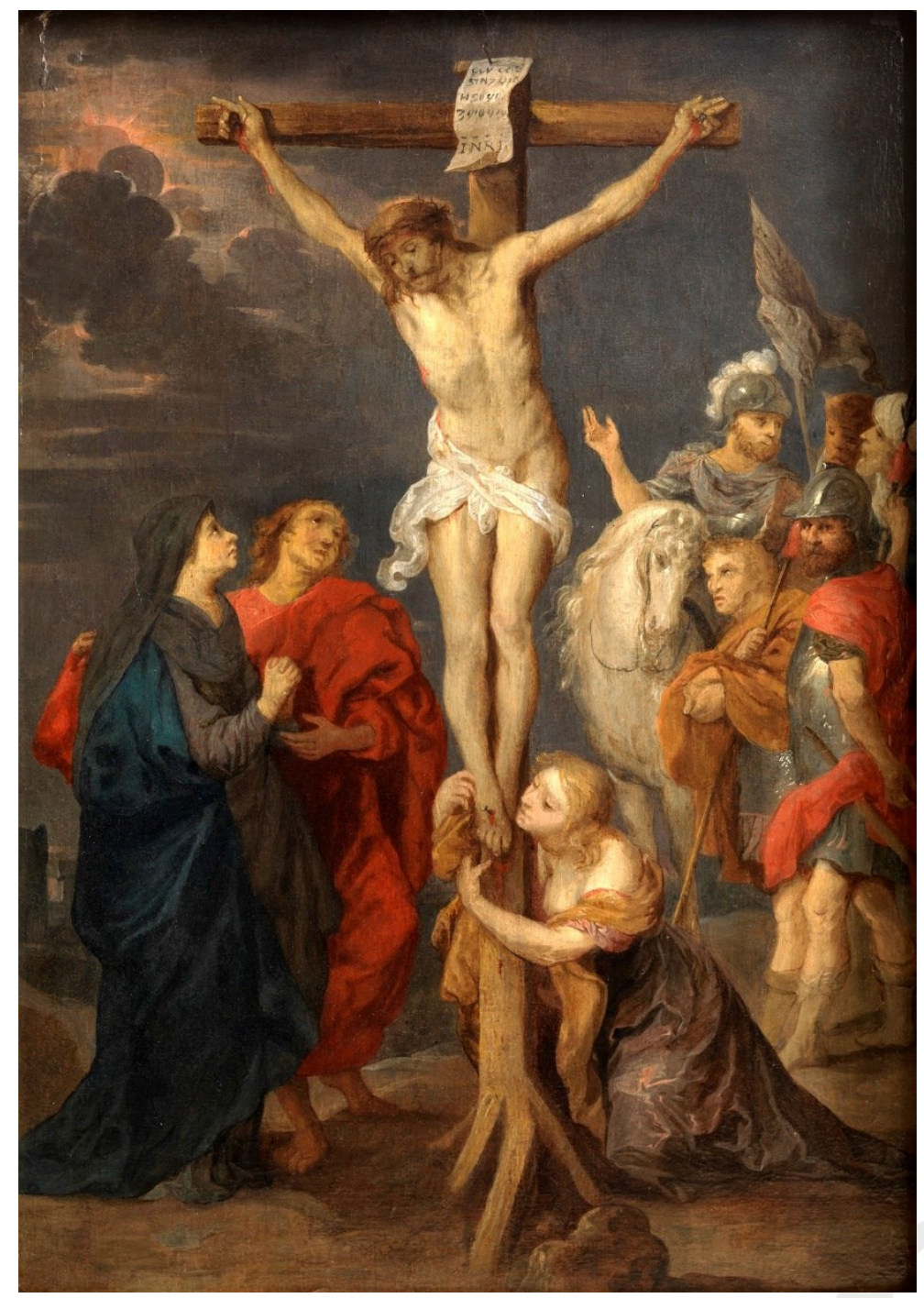

Fig. 2. Victor Wolfvoet, Crucifixión, colección Gerstenmaier, Madrid

Aquí, de pie, la Virgen y San Juan dirigen una implorante mirada hacia las alturas, reprimiendo las lágrimas y disponiendo sus manos en actitud de súplica ante la inevitable pérdida de Jesús. Éste, clavado en la cruz durante el sombrío atardecer que narran los evangelios, domina el eje de la composición, junto con la Magdalena de rubios cabellos y arrodillada en el suelo que, desolada, abraza y besa los pies de Cristo. Tras ella y algo distanciados, se sitúan los soldados antes referidos. Un oficial, vestido con armadura, capa roja y portando una lanza, interpela con su mirada directamente al espectador, de manera que se mantiene ajeno al suplicio que acontece en su presencia. Le acompaña otro soldado apoyado en una vara y 
desprovisto de casco, indicio de su inferior rango. Ya, al fondo, un centurión en su montura alza la mano derecha hacia Jesús agonizante en la cruz, al mismo tiempo que dirige unas palabras y gira el rostro hacia una figura masculina, con barba canosa y turbante.

Las diferencias entre ambas pinturas se reducen a escasos pormenores que

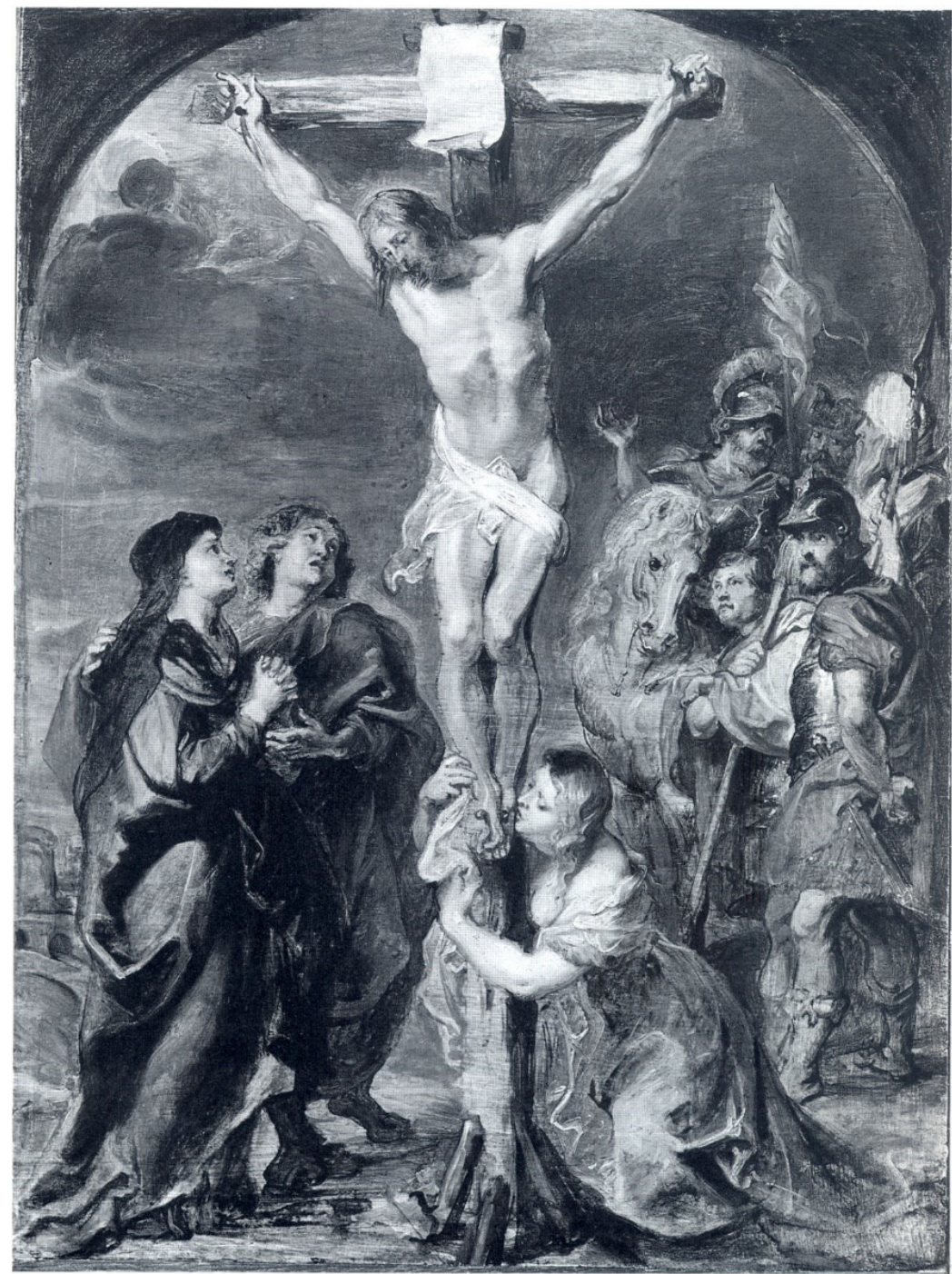

Fig. 3. Peter Paul Rubens, Crucifixión, Rockoxhuis, Amberes

en nada rompen con la concepción plástica del conjunto. Entre ellas, destaca la mano del centurión a caballo, más abierta y separada del cuerpo de Cristo en la réplica aquí tratada, en la que también es más contrastada y efectista la luz que baña el pecho y los muslos del crucificado.

El fundamento de esta composición se encuentra en el boceto de Peter Paul Rubens actualmente conservado en el Rockoxhuis de Amberes (Fig. 3) ${ }^{4}$, que

${ }^{4}$ Amberes, Rockoxhuis, (inv. no 34). Jay Richard Judson, The Passion of Christ, vol. VI, Corpus Rubenianum, (Harvey Miller Publishers, Turnhout, 2000), p. 133, no 34, fig. 104. 
sirvió de modelo para el grabado de Jacob Neeffs (Fig. 4$)^{5}$, cuya inscripción al pie "ECCE MATER TUA" alude al diálogo de Cristo con San Juan y María (Jn 19, 25-27), hecho que se plasma en esta escena. Este grabado es el que consideramos la fuente más directa de la pintura aquí estudiada, donde Victor Wolfvoet estilizó las figuras, amplió la longitud de la cartela que pende de lo alto de la cruz y agregó un texto en la misma, en contraposición al diseño de Rubens, cuyas figuras son más robustas y la cartela, más corta y sin inscripción.

El boceto de Rubens difiere de las réplicas y del grabado en el arco de medio punto que enmarca la zona superior de la composición y por la mayor prestancia de los protagonistas del drama. Por su parte, Victor Wolfvoet logró una bella armonía de contraste entre colores cálidos y fríos exquisitamente integrados en el espacio, aunque con más énfasis en los azules, los rojos, el verde esmeralda, el blanco y el ocre en las carnaciones. El discípulo siguió fiel al sentimiento de la contrarreforma que el maestro impuso en los Países Bajos $^{6}$.

La dependencia al boceto de Rubens es más próxima en la pintura de la colección Gerstenmaier, aunque ambas réplicas siguen prácticamente en todo al maestro. Pensamos afortunados los cambios de Victor Wolfvoet en el efectismo escenográfico de esta nueva réplica que se estudia. Así, la penumbra que envuelve a los soldados les resta protagonismo en favor de la figura de Jesús agonizante, aún más destacada por la incidencia directa de la luz en su cuerpo casi desnudo, como ya se señaló líneas atrás. No obstante, esta versión está lejos de alcanzar el vigor de la factura y la riqueza cromática del boceto de Rubens.

Fuentes coetáneas nos hablan de una Crucifixión que Victor Wolfvoet regaló a su tía, Livine van Geemont ${ }^{7}$. Sería un pretencioso optimismo pensar que alguna de las réplicas conocidas por nosotros pudiera vincularse con ésta, pero es un hecho que el tratamiento de la tabla aquí estudiada es el más cuidado de todas ellas. A ello hay que añadir que la rica ornamentación y el dorado del marco prueban la estima de la que gozó esta obra por parte de quienes la poseyeron en el pasado.

Rubens no terminó el encargo previsto en el boceto de la Rockxhuis para la capilla de la Santa Cruz de San Miguel de Gante ${ }^{8}$. El proyecto se frustró al tener que viajar a Madrid en 1628, por razones de estado sobradamente

\footnotetext{
${ }^{5}$ Max Rooses, L'oeuvre de P.P. Rubens: histoire et description de ses tableaux et dessins, phototypies, II, (Anvers, 1888), p. 93, no 294.

6 John B. Knipping, Iconography of the Counter Reformation in the Netherlands. Heaven on earth, II, (De Graff, 1974), p. 246, nota 13.

7 Frans Jos van den Branden, Geschiedenis der Antwerpsche Schilderschool, 2 vols., (Antwerpen, 1883), p. 799.

8 Philippe Kervyn de Volkaersbeke, Les Églises de Gand, II, (Ghent, 1858), pp. 9293, cit. Judson, Passion of Christ, p. 136, nota 4.
} 
conocidas $^{9}$. Por ello, el encargo se trasladó a Van Dyck, quien siguió, en buena parte, la idea del maestro fijada en el boceto ${ }^{10}$. El éxito de la composición alcanzó a Victor Wolfvoet que sigue el modelo con absoluta fidelidad. El boceto de Rubens lo poseyeron Delacroix y George Sand, entre otros ${ }^{11}$. Nadie como Victor Wolfvoet fue tan cercano a este boceto de Rubens para revivir aquel frustrado proyecto, aunque se sirvió para ello del grabado de Jacob Neefs $^{12}$. El éxito de esta composición lo prueban las numerosas copias de las que fue objeto ${ }^{13}$.

La atribución de la tabla que ahora tratamos a Abraham van Diepenbeeck, en su venta en París en 2008, es evidencia de su alta calidad, pues este otro discípulo de Rubens es uno de los más brillantes entre la pléyade de pintores de Amberes a mediados del siglo XVII pero, desgraciadamente, poco conocido en el coleccionismo español ${ }^{14}$.

Nos sentimos obligados a añadir una escueta nota bibliográfica al arte y personalidad de Victor Wolfvoet, puesto que ha sido un pintor escasamente conocido hasta fechas muy recientes. Los primeros artículos alusivos a sus obras fueron publicados en España ${ }^{15}$. Estas notas vienen a ratificar la enorme

9 Gregorio Cruzada Villaamil, Rubens, diplomático español: sus viajes a España y noticia de sus cuadros, (Casa Editorial de Medina y Navarro, 1874); Alejandro Vergara, Rubens and his Spanis Patrons, (Cambridge-Nueva York: University Press, 1999).

10 Susan J. Barnes et al., Van Dyck: A Complete Catalogue of the Paintings, (New Haven-London: Yale University Press, 2004), p. 263, nº III. 24.

11 Judson, Passion of Christ, p. 133, no 34.

12 Carl Gottfried Voorhelm Schneevoogt, Catalogue des estampes gravées d'après P.P. Rubens avec I'indication des collections où se trouvent les tableaux et les gravures, (Harlem, 1873), p. 47, no 324 y 325.

13 Judson, Passion of Christ, pp. 133-134.

14 Marie-Louise Hairs, Dans le sillage de Rubens. Les peintres d'histoire anversois au XVIIe siècle, (Liége: Université de Liège, 1977), pp. 151-181; David Wilton Steadman, Abraham van Diepenbeeck: Seventeenth Century Flemish Painter, (Michigan: UNI Research Press, 1982); con reseña de D. B. Hensbroeck-vam der Poel, Oud Holland, 100, 3-4, (1986), pp. 206-208.

15 Matías Díaz Padrón, "Tres cobres restituidos a Victor Wolfvoet, el más fiel seguidor de Rubens", Archivo Español de Arte, no 316, (2006), p. 407; Matías Díaz Padrón, "Dos cobres de Victor Wolfvoet en el Museo San Carlos de México", Boletín del Seminario de Estudios de Arte y Arqueología, no 65, (1999), p. 323; Matías Díaz Padrón, "Una adoración de los reyes de Victor Wolfvoet en el coleccionismo madrileño", Tendencias del Mercado del Arte, no 18, (2008), p. 32; Matías Díaz Padrón, "Tres nuevos cobres de Victor Wolfvoet con la Paz y la Guerra bajo la consigna de Rubens", Archivo Español de Arte, LXXXV, (2012), p. 88; Matías Díaz Padrón, "Una Andrómeda con Perseo y Pegaso en la lejanía de Victor Wolfvoet atribuida a David Teniers en la galería Christie's de Nueva York", Tendencias del Mercado del Arte, no 53, (2012), p. 92; Matías Díaz Padrón, "Un Calvario de Victor Wolfvoet en la colección Gerstenmaier", Tendencias del Mercado del Arte, no 68, (2013), p. 82. Recientemente Gregory Martin y Bert Schepers han tratado la obra de este pintor a partir de los estudios publicados en Archivo Español de Arte: Gregory Martin y Bert Schepers "Two Antwerp cabinets decorated by Victor Wolfvoet II", The Burlington Magazine, (2016), pp. 793-802. Mucho de lo publicado en este último artículo está registrado en el Archivo del Instituto Moll con la idea de un estudio más 
popularidad de su trabajo en la Península Ibérica, quizá por su cercanía al espíritu de Rubens, el cual, Wolfvoet, sabe trasmitir en sus obras, enlazando así con el gusto que el maestro flamenco supo fraguar en el público hispano.

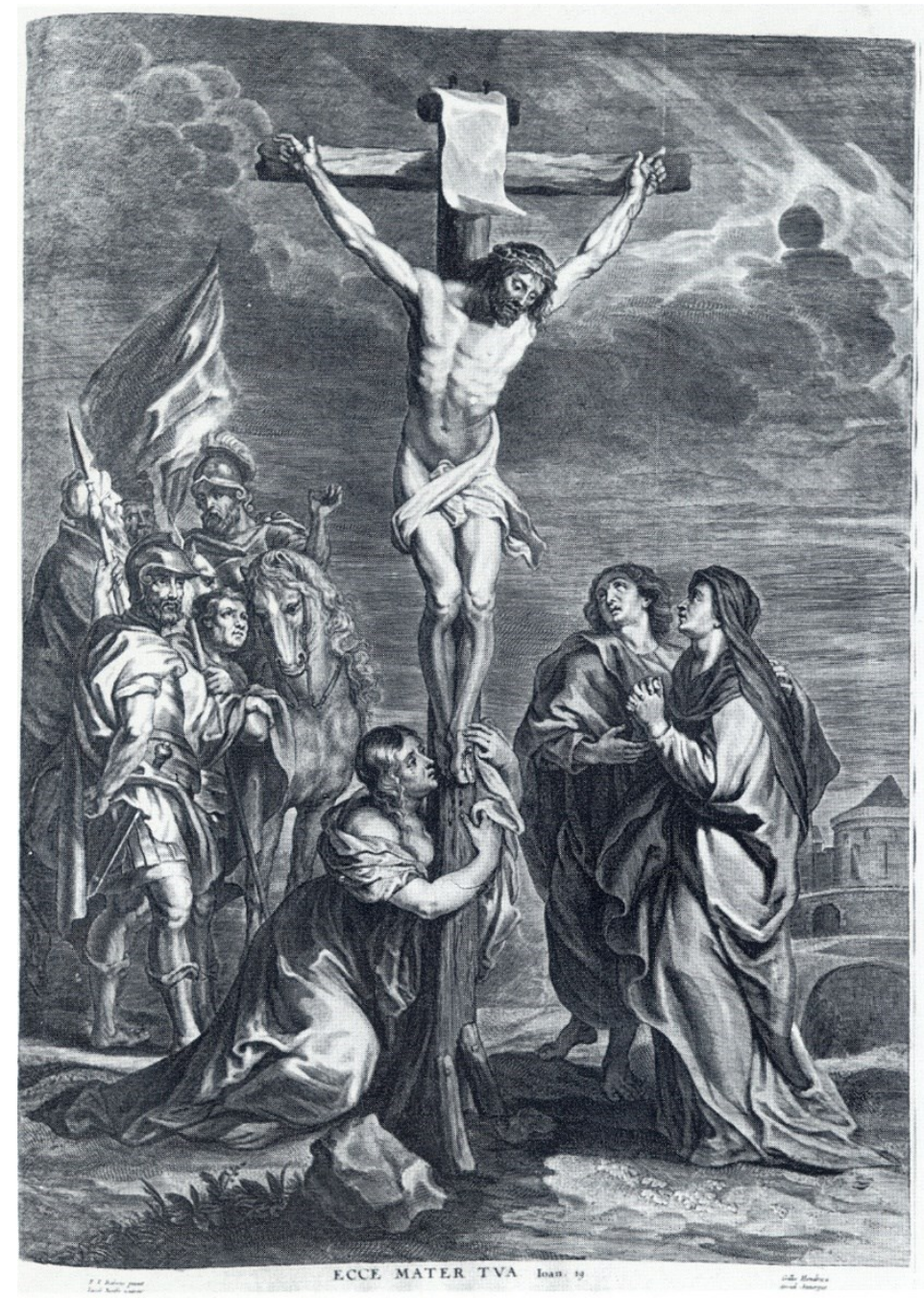

Fig. 4. Jacob Neefs siguiendo a Rubens, Crucifixión, grabado

Por otro lado, la Visitación de la iglesia de Santiago de Amberes, siempre atribuida a Victor Wolfvoet, la hemos restituido a Simon de Vos, demostrando el trabajo de este pintor en composiciones de gran formato ${ }^{16}$. Este error de identificación de la Visitación en la iglesia amberina, por espacio de cuatro siglos es la razón que impidió el conocimiento del estilo del pintor objeto de nuestro estudio.

completo de la obra de Victor Wolfvoet. No obstante, algunas más podemos añadir en el coleccionismo español, que esperamos publicar en el futuro.

16 Matías Díaz Padrón, "La Visitación de Saint-Jacques de Amberes: ¿Victor Wolfvoet o Simon de Vos?", Philostrato, Revista de Historia y Arte, no 1 (2017), pp.43-55. 
Bibliografía:

Alonso Blázquez 2017: Inmaculada Alonso Blázquez, "Victor Wolfvoet, Calvario" en Colección Hans Rudolf Gerstenmaier. La Pintura Flamenca en la Colección Gerstenmaier, dir. Matías Díaz Padrón y María Toral Oropesa, (Madrid: Lerner \& Tf Editores, 2017), pp. 82-83.

Barnes, De Poorter, Millar, Vey 2004: Susan J. Barnes, Nora de Poorter, Oliver Millar, Horst Vey, Van Dyck: A Complete Catalogue of the Paintings, (New Haven-London: Yale University Press, 2004).

Cruzada Villaamil 1874: Gregorio Cruzada Villaamil, Rubens, diplomático español: sus viajes a España y noticia de sus cuadros, (Casa Editorial de Medina y Navarro, 1874).

Díaz Padrón 1999: Matías Díaz Padrón, "Dos cobres de Victor Wolfvoet en el Museo San Carlos de México", Boletín del Seminario de Estudios de Arte y Arqueología, 65, (1999), pp. 323-328.

Díaz Padrón 2006: Matías Díaz Padrón, "Tres cobres restituidos a Victor Wolfvoet, el más fiel seguidor de Rubens", Archivo Español de Arte, 316, (2006), pp. 403-411.

Díaz Padrón 2008: Matías Díaz Padrón, "Una adoración de los reyes de Victor Wolfvoet en el coleccionismo madrileño", Tendencias del Mercado del Arte, 18, (2008), pp. 32-33.

Díaz Padrón 2012: Matías Díaz Padrón, "Tres nuevos cobres de Victor Wolfvoet con la Paz y la Guerra bajo la consigna de Rubens", Archivo Español de Arte, LXXXV, (2012), pp. 88-94.

Díaz Padrón 2012: Matías Díaz Padrón, "Una Andrómeda con Perseo y Pegaso en la lejanía de Victor Wolfvoet atribuida a David Teniers en la galería Christie's de Nueva York", Tendencias del Mercado del Arte, 53, (2012), pp. 92-94.

Díaz Padrón 2013: Matías Díaz Padrón, "Un Calvario de Victor Wolfvoet en la colección Gerstenmaier", Tendencias, 68, (2013), pp. 82-84.

Díaz Padrón 2017: Matías Díaz Padrón, "La Visitación de Saint-Jacques de Amberes: ¿Victor Wolfvoet o Simon de Vos?", Philostrato, Revista de Historia y Arte, 1, (2017), pp. 43-55.

Hairs 1977 : Marie-Louise Hairs, Dans le sillage de Rubens. Les peintres d'histoire anversois au XVIIe siècle, (Liége: Université de Liège, 1977). 
Hensbroeck-Van der Poel 1986 : Reseña de D. B. Hensbroeck-van der Poel, "David Wilton Steadman, Abraham van Diepenbeeck: Seventeenth Century Flemish Painter", Oud Holland, 100, 3-4, (1986), pp. 206-208.

Judson 2000: Jay Richard Judson, The Passion of Christ, vol. VI, Corpus Rubenianum, (Turnhout: Harvey Miller Publishers, 2000).

Knipping 1974: John B. Knipping, Iconography of the Counter Reformation in the Netherlands. Heaven on earth, II, (De Graff, 1974).

Martin, Schepers 2016: Gregory Martin, Bert Schepers "Two Antwerp cabinets decorated by Victor Wolfvoet II", The Burlington Magazine, (2016), pp. 793802.

Rooses 1888: Max Rooses, L'oeuvre de P.P. Rubens: histoire et description de ses tableaux et dessins, phototypies, II, (Anvers, 1888).

Schneevoogt 1873 : Carl Gottfried Voorhelm Schneevoogt, Catalogue des estampes gravées $d$ 'après P.P. Rubens avec I'indication des collections où se trouvent les tableaux et les gravures, (Harlem, 1873).

Steadman 1982: David Wilton Steadman, Abraham van Diepenbeeck: Seventeenth Century Flemish Painter, (Michigan: UNI Research Press, 1982).

Van den Branden 1883: Frans Jos van den Branden, Geschiedenis der Antwerpsche Schilderschool, 2 vols., (Antwerpen, 1883).

Vergara 1999: Alejandro Vergara, Rubens and his Spanis Patrons, (Cambridge-Nueva York: University Press, 1999).

Volkaersbeke 1858: Philippe Kervyn de Volkaersbeke, Les Églises de Gand, II, (Ghent, 1858). 